\title{
Squeeze-Out of Branched Alkanes on Graphite
}

\author{
N. N. Gosvami and S. K. Sinha \\ Department of Mechanical Engineering, National University of Singapore, 9 Engineering Drive 1, Singapore 117576
}

\author{
S. J. O'Shea*
}

Institute of Materials Research and Engineering, 3 Research Link, Singapore 117602

(Received 16 August 2007; revised manuscript received 10 January 2008; published 20 February 2008)

\begin{abstract}
We study squalane and heptamethylnonane (HMN) confined between a conducting atomic force microscope tip and a graphite surface. Solvation layering occurs for both liquids but marked differences in the squeeze out mechanics are observed for ordered or disordered monolayers. The squalane monolayer at $25^{\circ} \mathrm{C}$ is an ordered solid, as verified by direct imaging, and the squeeze out can be modeled using elastic continuum mechanics. $\mathrm{HMN}$ is in a disordered state at $25^{\circ} \mathrm{C}$ and cannot be modeled as a single elastic asperity even in solid-solid contact because HMN liquid is trapped in the contact zone.
\end{abstract}

DOI: 10.1103/PhysRevLett.100.076101

Liquids confined between two surfaces can form ordered layers, which may lead to oscillatory solvation forces [1]. These forces arise from the variation in the molecular density of the liquid between the surfaces. For geometric reasons, the liquid molecules confined between the surfaces may pack (or order) into well-defined solvation layers. The state of the confined material (e.g., solid- or liquid-like) strongly influences how a particular liquid is squeezed out from the two approaching surfaces and is thus critical in understanding lubrication, adhesion, and wear [2-4].

One key parameter affecting the material state is the shape of the molecules. Specifically, various simulations and experimental studies have studied linear and branched alkanes [4-19]. Many linear alkane molecules reveal strong solvation layering [5]. Studies on branched alkanes remain controversial. Early research suggested there was no solvation layering for branched molecules [6,7], but more recent simulations [4,8-14] and experimental studies [15-19] show that this is not necessarily the case. However, if such solvation layers do occur, the confined molecules tend to be more "liquid-like" in comparison to corresponding linear alkanes due to poorer inplane ordering $[4,9-11,13,14]$. A more liquid like state arising from increased branching can also dramatically affect the squeeze out behavior of the confined fluid, with the squeeze out of the last layer becoming sluggish, which leads to trapping of molecules within the contact zone [4].

Many experiments studying branched molecules use squalane $\quad(2,6,10,15,19,23$-hexamethyltetracosane $), \quad$ a model boundary lubricant [15-19]. Measurements indicate strong solvation layering of squalane close to various solid surfaces, with the molecules lying parallel to the surface. However, previous experimental studies conclude that there is no in-plane ordering. Our results for squalane on graphite show that this is not the case. Specifically, we study, using atomic force (AFM) and scanning tunneling microscopy (STM), the branched liquids squalane and
PACS numbers: 68.08.-p, 46.55.+d, 68.37.Ps, 68.43.Hn

2,2,4,4,6,8,8-heptamethylnonane (HMN) near a graphite surface. We present the first direct imaging of surface ordering in a branched alkane (i.e., squalane), which is in agreement with simulations [20]. In contrast, HMN remains disordered on the surface. This disorder/ordered distinction in the state of the confined monolayer leads to striking differences in the solvation layering and squeeze out behavior. The squeezing of the confined squalane monolayer shows solid-like behavior and can be described by a continuum mechanics model. Squeezing of HMN reveals significant variability in data due to its disordered state, and simple, continuum models of an elastic contact are found to be inapplicable.

The AFM and STM experiments were performed on a commercial instrument (Molecular Imaging). Conduction AFM (C-AFM) was used for simultaneous force and conductivity measurements. Rectangular $\mathrm{Si}_{3} \mathrm{~N}_{4}$ cantilevers (Olympus, ORC8-PS-W) with normal spring constant of $0.76 \mathrm{~N} / \mathrm{m}$ were used. The cantilevers were coated with $5 \mathrm{~nm} \mathrm{Cr}$ and $\sim 35 \mathrm{~nm}$ Au to make conducting. Current flow between the AFM tip and sample was measured from the tip using a Kiethly amplifier (model 6485). The bias voltage was applied to the sample, and a fixed resistor $(40 \mathrm{k} \Omega)$ was placed in series to limit the current flow. Experiments were performed on freshly cleaved highly oriented pyrolytic graphite (HOPG). For STM experiments, a drop of pure squalane or HMN liquid (Sigma-Aldrich, as purchased $\geq 98 \%$ ) was placed on the HOPG and the tip immersed in the drop. Mechanically cut Pt/Ir wires were used. For AFM experiments, the cantilever and graphite surface were completely immersed in a Teflon liquid cell containing squalane or HMN. The main AFM data obtained consist of force curves, in which the deflection of a cantilever is measured as the tip-to-sample distance is changed. The cantilever deflection can be converted to a force by multiplying by the cantilever spring constant. In C-AFM experiments, the current flow with a fixed bias voltage is measured simultaneously with the force curve acquisition. After experiments, the tips were imaged by 
scanning electron microscopy (SEM) to estimate the tip radius of curvature.

Figure 1 shows representative force curves taken in squalane and $\mathrm{HMN}$ at $25^{\circ} \mathrm{C}$. For squalane, $\sim 5$ solvation jumps were clearly observed as indicated by the labels $n=$ $1-5$, where $n=0$ corresponds to the tip in direct contact with the graphite substrate, and $n=1$ is the monolayer. In HMN, we observe weaker solvation layering (labeled $n=$ 1-3), and most of the force curves do not reveal sharp jumps between layers. The transition between solvation layers resembles that of yielding of a polymer monolayer [21], with small kinks (shown by unlabelled arrows) observed in the layer closest to the graphite surface $(n=1)$ suggesting a change in conformation of the confined molecules under compression.

We first consider the squalane data. Several current vs force curves obtained at $25^{\circ} \mathrm{C}$ using the same tip on the first monolayer $(n=1)$ are shown in Fig. 2. All the curves show similar variation with load, although the force needed to squeeze out a layer (i.e., the $n=1 \rightarrow 0$ and $n=2 \rightarrow 1$ transition) varies slightly. The curves reveal two distinct regions; where the current shows a slow rise with force at low forces and a fast current increase close to the complete squeeze out of the monolayer $(n=1 \rightarrow 0)$. This behavior is similar to that observed for a hexadecane monolayer on HOPG [22]. We can model the slow varying region using continuum elastic mechanics, assuming the current is proportional to the tip-sample contact area [22]. The inset (Fig. 2) shows the current variation as the tip is pulled off the squalane monolayer $(n=1)$. The data is well fitted by the Derjaguin-Muller-Toporov (DMT) model, and the contact radius can be written [23],

$$
a^{2}=\left\{\frac{R}{K}\left(F+F_{c}\right)\right\}^{2 / 3}=A I
$$

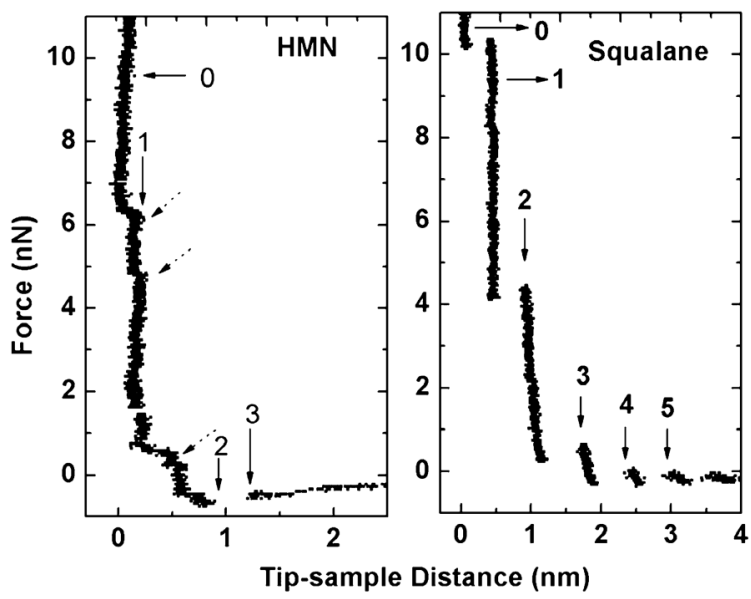

FIG. 1. Data showing force as a function of the tip-sample separation for squalane and HMN on HOPG. Clear solvation jumps are observed in squalane indicated by $n=0-5$ ( $n=0$ is the graphite surface). HMN shows less defined jumps indicated by $n=0-3$ ( $n=0$ is the graphite surface) with several kinks in the force curve (shown with unlabelled arrows). where $A$ is a proportionality constant, $I$ is the measured current, $R$ is the tip radius of curvature, $F$ is the applied force, $F_{c}$ is the pull-off force, and $K$ is the effective modulus for the gold-HOPG system (39.5 GPa [22]).

The good fit of the current vs force data to the DMT model shows that in the slow varying current region, change in the tip-sample contact area is the principal cause of the observed variation in current flow. This is entirely similar to observations in hexadecane [22] and indicates that squalane also behaves as an elastic, solid-like monolayer at $25^{\circ} \mathrm{C}$. We confirm this by STM imaging of the squalane monolayer, which reveals ordered domains of lamellar structures [Fig. 3(a)]. Molecular resolution images reveal individual squalane molecules aligned parallel to each other [Fig. 3(b)]. These are the first images of squalane and support recent simulations of such surface ordering [20]. These authors also showed that previous experimental studies based on diffraction methods $[15,17]$ are unable to confirm ordering in squalane due to broadening of the diffraction peaks. The lamellar spacing is $\sim 4.0 \mathrm{~nm}$, and we observe a diffuse boundary between the squalane lamellar stripes. This contrasts with linear alkanes such as hexadecane and tetracosane (a molecule of similar chain length to squalane), where very sharp lamellar boundaries are observed [24].

We now compare force curve data of HMN with squalane to reveal fundamental differences between the two confined fluids. Figure 4 shows current vs force measurements at $25^{\circ} \mathrm{C}$ where the tip is probing the HMN monolayer $(n=1)$. Compared to squalane, the current signals do not show a systematic variation with force; the squeeze out force for the $n=1 \rightarrow 0$ transition varies considerably (by a factor of 2); and the data cannot be fitted to any generalized model of an elastic point contact, such as the Maugis-Dugdale model [23].

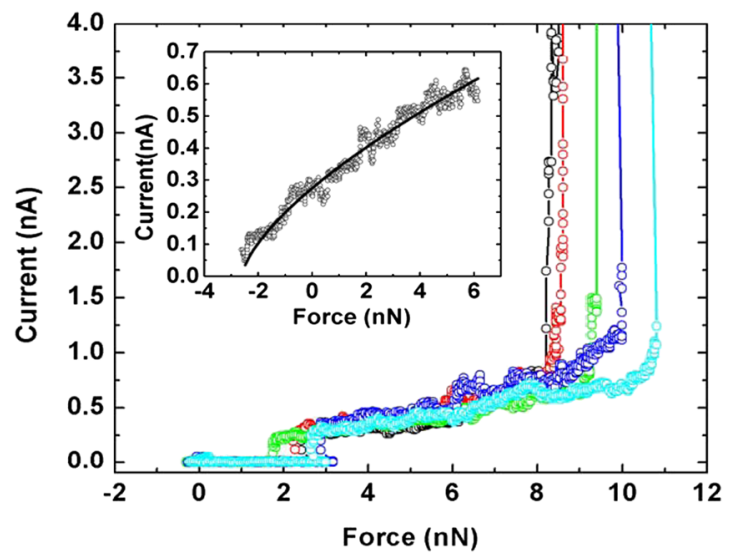

FIG. 2 (color online). Current vs force curve for the tip in contact with the first squalane layer $(n=1)$. There are distinct "slow varying current" and "fast varying current" regions (the latter being close to the $n=1 \rightarrow 0$ layer transition at $8-10 \mathrm{nN}$ force). The inset shows data, taken with the same tip, as the tip is pulled off the first layer. The variation of current with force follows a DMT model. 
(a)

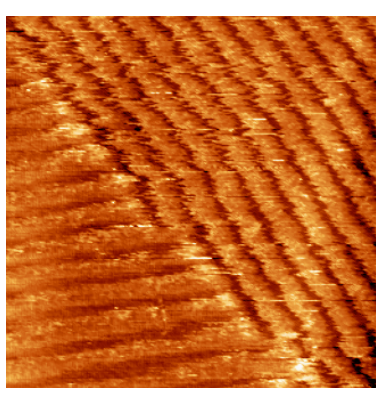

(b)

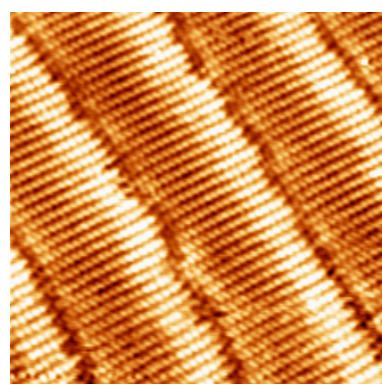

FIG. 3 (color online). (a) STM topographic image of the squalane monolayer on HOPG showing ordered domains. Image size $=60 \mathrm{~nm} \times 60 \mathrm{~nm}$. Tunnel conditions: $V=$ $600 \mathrm{mV}$ (sample positive), $i_{t}=10 \mathrm{pA}$. (b) Molecular resolution STM topographic image revealing individual squalane molecules. Image size $=13 \mathrm{~nm} \times 13 \mathrm{~nm}$. Tunnel conditions: $V=$ $1.0 \mathrm{~V}$ (sample positive), $i_{t}=12 \mathrm{pA}$.

Figure 5 shows current vs force data for squalane and HMN with the tip in contact with the HOPG substrate $(n=0)$. In squalane at $25^{\circ} \mathrm{C}$ [Fig. 5(c)], the current vs force curves are reversible in loading and unloading cycles. Assuming the measured current is proportional to contact area, we find the DMT model [Eqn. (1)] again provides a good fit to the data. Thus, as shown previously for contacts in hexadecane [22], the relative change in contact area of the tip-HOPG junction is well described by an elastic continuum model. The tip-HOPG contact mechanics is very different in HMN [Fig. 5(a)]. We observe a gradual variation of current, even up to forces of $\sim 20 \mathrm{nN}$ where the junction resistance in HMN $\left(137 \pm 65 \mathrm{M} \Omega\right.$ per nm $\left.{ }^{2}\right)$ approaches the resistance found in squalane (77 \pm $21 \mathrm{M} \Omega$ per $\mathrm{nm}^{2}$ ), an observation which gives confidence that some part of the tip is indeed in contact with the HOPG surface. The current variation with load cannot be fitted to elastic contact models [23] and does not follow a reversible path. Significant hysteresis and variability is observed between the approach and retraction cycles.

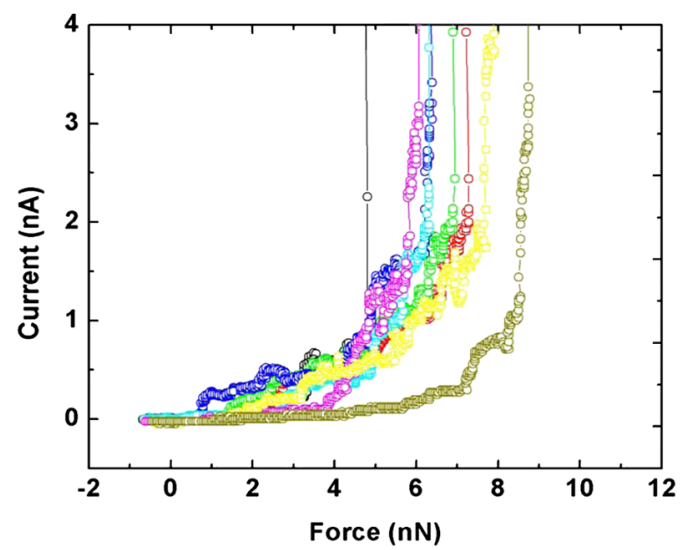

FIG. 4 (color online). Current vs force curve for HMN at low force. The tip is within the monolayer $(n=1)$ over most of this force region (there is uncertainty at the higher forces on whether the tip is within $n=1$ or $n=0$ ). The current variation with force is highly variable.

We believe the trends in the HMN data (hysteresis, nonuniform variation with force) arise because the HMN molecules are in a liquid-like state. It is significant that we cannot fit elastic continuum models to the current-force data for both the HMN monolayer $(n=1)$ of Fig. 4 and to the tip-HOPG junction $(n=0)$ of Fig. 5. This result is surprising and implies that the increased fluidity of the material near or within the junction negates the use of point contact models developed for simple elastic solids.

Strong support that the change in the state of the confined material (liquid-like or solid-like) is the underlying cause is given by experiments at different temperatures. Figure 5(b) shows a representative force curve undertaken in squalane at $65{ }^{\circ} \mathrm{C}$ (note: the entire liquid cell is isothermal), with the tip in mechanical contact with the HOPG substrate $(n=0)$. The force curve behavior resembles that of HMN at $25^{\circ} \mathrm{C}$ [Fig. 5(a)]. Further, despite repeated attempts, no STM image of the squalane monolayer at $65^{\circ} \mathrm{C}$ could be obtained, strongly suggesting the monolayer has ceased to be ordered. This assertion is supported by a recent simulation [20], which shows that the solid phase of the squalane monolayer on graphite melts at $\sim 52^{\circ} \mathrm{C}$. Similarly, we have observed that the hexadecane monolayer on graphite is well ordered at $25^{\circ} \mathrm{C}$ and shows solid-like squeeze out [22], whereas at $65^{\circ} \mathrm{C}$, the monolayer cannot be imaged and shows force curves similar to Fig. 5(b). The hexadecane monolayer on graphite melts at $\sim 55^{\circ} \mathrm{C}$ [25].

Thus, the state of the confined material, not the branching, is the key condition dictating the squeeze out behavior and dynamics [26]. The degree of branching and temperature influence the specific state of the monolayer. Note that HOPG is not a special substrate for the formation of ordered molecular layers. Surface flatness is more important, and the HOPG lattice has little effect on the surface ordering of alkane monolayers [27]. Linear alkanes also form ordered monolayers on a variety of other substrates having significant lattice mismatch, e.g., $\mathrm{MoS}_{2}, \mathrm{MoSe}_{2}$, $\mathrm{Au}(111)[27,28]$.

An explanation of the differences in the observed force curves [e.g., between Fig. 5(a) and 5(c)] can be found in recent simulations comparing the squeeze out of a linear alkane (butane) and its branched isomer, isobutane [4]. The linear molecules form an ordered monolayer and are completely removed from the contact zone under applied pressure. The branched isomer (isobutane) remains liquid-like in the contact zone under identical conditions of temperature and pressure and shows a higher resistance to displacement, leading to the trapping of a few molecules, even at very high pressure [4]. Essentially, the confined molecules display viscoelastic behavior and are displaced only slowly from the gap. If the pressure becomes sufficiently high before the molecules can be displaced, then the confining surfaces will deform enabling hollows filled with isobutane to be created. We confirm this interpretation by drifting the tip extremely slowly $(\sim 1.0 \mathrm{~nm} / \mathrm{sec})$ towards the surfaces in $\mathrm{HMN}$; waiting $\sim 10$ seconds at high applied 


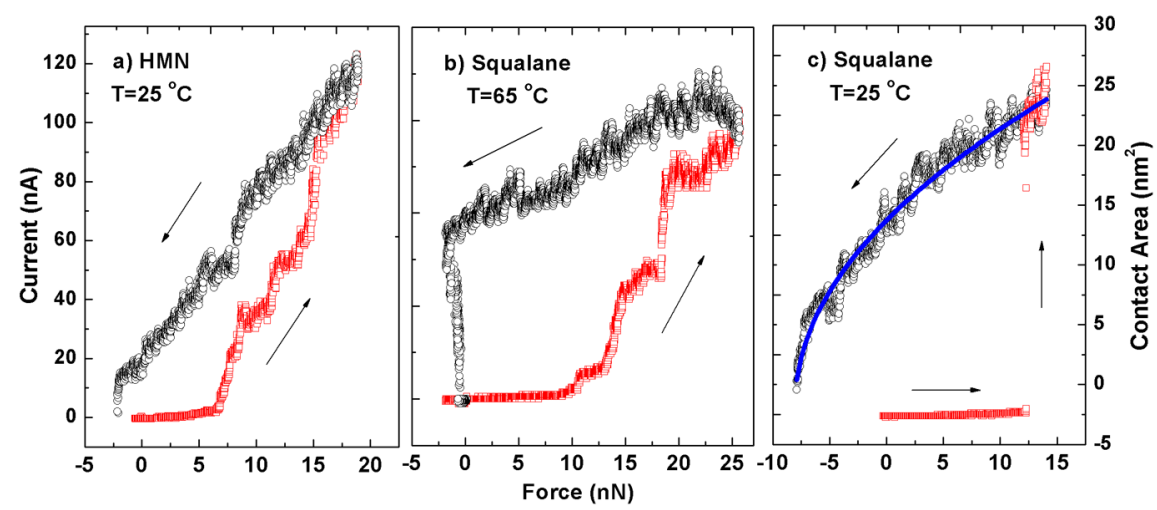

FIG. 5 (color online). Current vs force curve at high force. The arrows indicate the direction of the force curve cycle. The tip-sample approach speed is $10 \mathrm{~nm} / \mathrm{s}$. (a) Data for $\mathrm{HMN}$ at $25^{\circ} \mathrm{C}$. The curve does not show a sharp squeeze out of the monolayer $(n=1 \rightarrow 0$ transition), although consideration of the current magnitude indicates that some part of the tip apex is in contact with HOPG at forces $\geq 10 \mathrm{nN}$. (b) Data for squalane at $65^{\circ} \mathrm{C}$. The curve does not show a sharp squeeze out of the monolayer $(n=1 \rightarrow 0$ transition) and cannot be fitted to a continuum elastic model. (c) Data for squalane at $25^{\circ} \mathrm{C}$. The monolayer is squeezed out suddenly $(n=1 \rightarrow 0$ transition) and the tip contacts the HOPG when the force reaches $\sim 12.5 \mathrm{nN}$ (squares). The variation in current while pulling the tip off the HOPG surface (circles) is fitted with the DMT model (solid curve) to give the contact area.

load; and finally pulling the tip off the surface as in a routine force curve. We observe a force curve which is similar to the pull-off curve in Fig. 5(c), demonstrating that a solid-solid contact can be formed in HMN if the loading rate is very slow. Thus, trapping of HMN molecules under the tip during compression appears a plausible mechanism.

In conclusion, we observe that a branched alkane (squalane) can form ordered monolayers on graphite at $25^{\circ} \mathrm{C}$. Conduction AFM data shows that the expulsion of the ordered squalane molecules is a step-like event and resembles that of a known solid-like monolayer, namely, hexadecane [22]. Continuum elastic models are well suited to describing the current-force data of ordered, solid-like material such as squalane and hexadecane. However, when the confined monolayer is disordered, as for HMN or squalane and hexadecane at $65^{\circ} \mathrm{C}$ (i.e., above the monolayer melting temperatures), the current vs force curve is qualitatively very different, and simple elastic models cannot be applied at the approach speeds used because confined liquid molecules remain trapped within the junction.

*s-oshea@imre.a-star.edu.sg

[1] R. G. Horn and J. N. Israelachvili, J. Chem. Phys. 75, 1400 (1981).

[2] M. Y. He, A.S. Blum, and G. Overney et al., Phys. Rev. Lett. 88, 154302 (2002).

[3] T. Schmatko, H. Hervet, and L. Leger, Phys. Rev. Lett. 94, 244501 (2005).

[4] U. Tartaglino, I. M. Sivebaek, and B. N. J. Persson et al., J. Chem. Phys. 125, 014704 (2006).

[5] H. K. Christenson, D. W. R. Gruen, and R. G. Horn et al., J. Chem. Phys. 87, 1834 (1987).

[6] J. N. Israelachvili, S. J. Kott, and M.L. Gee et al., Macromolecules 22, 4247 (1989).

[7] J. N. Israelachvili, S.J. Kott, and M.L. Gee et al., Langmuir 5, 1111 (1989).
[8] Y. T. Wang, K. Hill, and J. G. Harris, J. Chem. Phys. 100, 3276 (1994)

[9] C. J. Mundy, S. Balasubramanian, and K. Bagchi et al., Faraday Discuss. 104, 17 (1996).

[10] M. Dijkstra, J. Chem. Phys. 107, 3277 (1997).

[11] J. P. Gao, W. D. Luedtke, and U. Landman, J. Chem. Phys. 106, 4309 (1997).

[12] J. C. Wang and K. A. Fichthorn, J. Chem. Phys. 108, 1653 (1998).

[13] S. T. Cui, P. T. Cummings, and H. D. Cochran, J. Chem. Phys. 114, 6464 (2001).

[14] J. C. Wang and K. A. Fichthorn, J. Chem. Phys. 116, 410 (2002).

[15] D. Fuhrmann, A. P. Graham, and L. Criswell et al., Surf. Sci. 482, 77 (2001).

[16] R. Lim and S. J. O'Shea, Phys. Rev. Lett. 88, 246101 (2002).

[17] D. Fuhrmann and A. P. Graham, J. Chem. Phys. 120, 2439 (2004).

[18] Y.X. Zhu and S. Granick, Phys. Rev. Lett. 93, 096101 (2004).

[19] H. D. Mo, G. Evmenenko, and P. Dutta, Chem. Phys. Lett. 415, 106 (2005).

[20] A. D. Enevoldsen, F. Y. Hansen, and A. Diama et al., J. Chem. Phys. 126, 104703 (2007).

[21] S. J. O'Shea, M. E. Welland, and T. Rayment, Langmuir 9, 1826 (1993).

[22] N. N. Gosvami, S. K. Sinha, and W. Hofbauer et al., J. Chem. Phys. 126, 214708 (2007).

[23] R. W. Carpick, D. F. Ogletree, and M. Salmeron, J. Colloid Interface Sci. 211, 395 (1999).

[24] L. Askadskaya and J. P. Rabe, Phys. Rev. Lett. 69, 1395 (1992).

[25] P. Espeau and J. W. White, J. Chem. Soc., Faraday Trans. 93, 3197 (1997).

[26] A. D. Enevoldsen, F. Y. Hansen, A. Diama, et al., J. Chem. Phys. 126, 104704 (2007).

[27] S. Cincotti and J. P. Rabe, Appl. Phys. Lett. 62, 3531 (1993).

[28] H. M. Zhang, Z. X. Xie, B. W. Mao, et al., Chem. Eur. J. 10, 1415 (2004). 